\title{
Supermassive black holes in spiral galaxies: HST/STIS observations for three new objects
}

\author{
L. Coccato ${ }^{1}$, E. Dalla Bontà ${ }^{1}$, M. Sarzi ${ }^{2}$ A. Pizzella ${ }^{1}$, E. M. Corsini ${ }^{1}$ \\ and F. Bertola ${ }^{1}$ \\ ${ }^{1}$ Dipartimento di Astronomia, Università di Padova, Padova, Italy \\ ${ }^{2}$ University of Oxford, UK
}

\begin{abstract}
We present long-slit HST/STIS measurements of the ionized-gas kinematics in the nucleus of three disk galaxies, namely NGC 2179, NGC 4343, NGC 4435. The sample galaxies have been selected on the basis of their ground-based spectroscopy, for displaying a strong central velocity gradient for the ionized gas, which is consistent with the presence of a circum nuclear keplerian disk (CNKD, Bertola et al. 1998; Funes et al. 2002) rotating around a super massive black hole (SMBH). For each target galaxy we obtained the $\mathrm{H} \alpha$ and [NII] $6583 \AA$ kinematics along the major axis and two 0"' 25 parallel offset positions. Out of three objects only NGC 4435 turned out to have a disk of ionized gas in regular motion and a regular dust-lane morphology. Preliminary modeling indicates a SMBH mass $\left(M_{\bullet}\right)$ one order of magnitude lower than the one expected from the $M_{\bullet}-\sigma_{c}$ relation for galaxies (Ferrarese \& Merritt 2000; Gebhardt et al. 2000).
\end{abstract}

As demonstrated in Bertola et al. (1998), it is possible to detect from ground-based observations the presence of a CNKD around a SMBH. Its identification is possible from the study of the position velocity (PV) diagram as done by Funes et al. (2002). From this work and literature data we select three galaxies, namely NGC 2179, NGC 4343 and NGC 4435, which have a PV diagram consistent with the presence of a CNKD and a central stellar velocity dispersions $\left(\sigma_{c}\right)$ which falls in a poorly sampled region of the $M_{\bullet}-\sigma_{c}$ relation.

For each target galaxy we obtained with HST/STIS the H $\alpha$ and [NII] $6583 \AA$ kinematics along the major axis and two 0." 25 parallel offset positions on either sides of the nucleus. The scale was $0 . " 05$ pixel $^{-1}$, the instrumental dispersion was $0.55 \AA$ pixel $^{-1}(\simeq 25 \mathrm{~km}$ $\mathrm{s}^{-1}$ ) and the slit width was 0." 2 . In Fig. 1 we report the dust-lane morphology obtained by the unsharp-masked HST images and the STIS major-axis spectra of the three objects.

For NGC 2179 and NGC 4343 the spectrum reveals a disturbed gas kinematics. These two objects show an irregular dust-lane morphology, too. On the contrary, NGC 4435, the only one with a regular kinematics, shows also a regularity of the dust-lane morphology. This galaxy is a good candidate to model the velocity field for the determination of $M_{\mathbf{\bullet}}$. In the model we consider a constant velocity dispersion field, an exponential profile for the flux of the emission lines and we build the velocity field considering the contribution of the stellar potential (measured from the surface-brightness profile using a constant mass-to-light ratio and spherical symmetry) and the Keplerian potential of a SMBH. Effects of the STIS PSF, of the slit width and the bleeding of charge between adjacent pixels in the CCD are also taken into account.

We summarize our results as follows.

- Ground-based observations evidence that about $20 \%$ of galaxies show a PV diagram which is consistent with the presence of a CNKD (Funes et al. 2002). This criterion should be combined with the presence of a regular dust-lane morphology, according to the results 

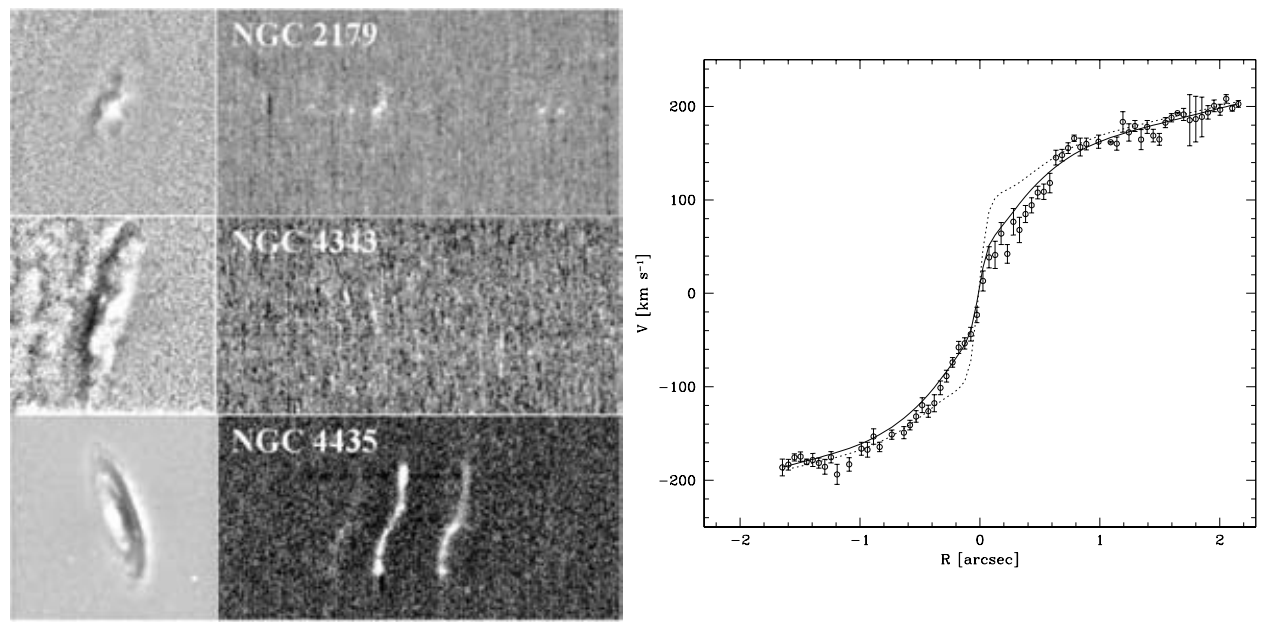

Figure 1. Left panel. Dust-lanes morphology obtained by the unsharped-masked HST images and STIS major-axis spectra; $\mathrm{H} \alpha$ and [NII] emission lines are clearly visible in NGC 4435. Right panel. Circles: observed rotation curve along the major axis of NGC 4435. Solid line: our model; dashed line: gas rotation curve assuming the $M_{\bullet}$ predicted by the $M_{\bullet}-\sigma_{c}$ relation $\left(M_{\bullet} \simeq 10^{7} M_{\odot}\right.$, $\sigma_{c}=174 \mathrm{~km} \mathrm{~s}^{-1}$ from Bernardi et al. 2002.)

later found by Ho et al. (2002). Indeed in our sample NGC 4435 is the only galaxy that shows a regular dust-lane morphology as well as a regular rotation curve (Fig. 1, left panel). Combining these two criteria we maximize the chances to select successful STIS target galaxies for gas-dynamical measurements of SMBH masses. On the basis of this experience we are tempted to conclude that only less than the $10 \%$ of galaxies shows a ground-based PV diagram which is consistent with the presence of a CNKD and has also a regular rotation curve, useful for the measurements of the SMBH mass in a HST/STIS spectroscopic follow-up.

- The modeling work is still in progress (Fig. 1, right panel), but preliminary results give a $M_{\bullet}$ about one order of magnitude lower than the $M_{\bullet}$ predicted by the $M_{\bullet}-$ $\sigma_{c}$ relation. We are going to include in our model also the available HST/STIS minor axis kinematics, and we are going to add an exponential velocity dispersion profile and evaluate the asymmetric drift correction, as done by Barth et al. (2001).

\section{References}

Barth, A. J., Sarzi, M., Rix, H.-W., Ho, L., C., Filippenko, A., V., \& Sargent, W., L. W. 2001, ApJ, 555, 685

Bernardi, M., Alonso, M. V., da Costa, L. N., Willmer, C. N. A., Wegner, G., Pellegrini, P. S., Rité, C., \& Maia, M. A. G. 2002, AJ, 123, 2990

Bertola, F., Cappellari, M., Funes, J. G., Corsini, E. M., Pizzella, A., \& Vega Beltrán, J. C. 1998, ApJ, 509, L93

Ferrarese, L. \& Merritt, D. 2000, ApJ, 539, L9

Funes, J. G., J., S., Corsini, E. M., Cappellari, M., Pizzella, A., Vega Beltrán, J. C., Scarlata, C., \& Bertola, F. 2002, A\&A, 388, 50

Gebhardt, K., et al. 2000, ApJ, 539, L13

Ho, L. C., Sarzi, M., Rix, H., Shields, J. C., Rudnick, G., Filippenko, A. V., \& Barth, A. J. 2002, PASP, 114, 137 